\title{
Tratamento cirúrgico das valvopatias. Parte 1
}

\author{
Domingo M. BRAILE* ${ }^{*}$ Marco A. VOLPE ${ }^{\star *}$, Serginando L. RAMIN ${ }^{\star \star *}$, Dorotéia R. S. SOUZA***
}

RBCCV 44205-239

BRAILE, D. M.; VOLPE, M. A.; RAMIN, S. L.; SOUZA, D. R. S. - Tratamento cirúrgico das valvopatias. Parte 1. Rev. Bras. Cir. Cardiovasc., 9 (2): 113-122, 1994.

RESUMO: Este assunto, de amplo aspecto, será abordado em três partes. O presente trabalho trata-se do histórico, indicação cirúrgica, conduta pré-operatória e próteses valvulares cardiacas. Os demais capítulos discutirão sobre técnica operatória, reoperaçōes e situaçōes especiais, como o tratamento cirúrgico na endocardite e experiências com válvula de pericárdio bovino.

DESCRITORES: valvas cardiacas, cirurgia; valvopatias, história; valvopatias, indicação cirúrgica; valvopatias, conduta clínica; próteses valvulares cardiacas.

\section{HISTÓRICO}

A conquista cirúrgica do coração foi feita de forma lenta e gradual, iniciada às custas de pioneiros idealistas, que contavam com o apoio de poucos e o descrédito de muitos. A cirurgia das valvas representou o início da cirurgia cardíaca e, por isso mesmo, foi a mais rica em episódios heróicos.

Para realizar a cirurgia valvar, o homem teve que conquistar primeiramente 0 direito de penetrar com os dedos ou instrumentos nas cavidades cardiacas e, depois, capacitar-se para substituir as funções do coração por um circuito mecânico, que permitisse a atuação a céu aberto sobre as valvas.

Há quase 100 anos, SUMWAIS ${ }^{61}$, em 1898 , foi o primeiro a sugerir a secção de uma valva mitral estenosada.

O resumo histórico da cirurgia valvar inicia-se nos trabalhos de DOYEN ${ }^{30}$, que, em 1913 , realizou o primeiro reparo cirúrgico em valva cardiaca humana, seccionando uma valva pulmonar estenosada.
No mesmo ano, Theodor TUFFIER 67 operou um homem com severa estenose aórtica, realizando uma dilatação digital. A primeira operação para estenose mitral foi feita por CUTTLER ${ }^{27}$, em 1923 , fazendo excisão de uma pequena porção da cúspide anterior da valva mitral com auxílio de um tenótomo.

Em 1925, SOUTTAR 66, introduzindo o dedo indicador através da aurícula esquerda, realizou a primeira dilatação digital da valva mitral.

No final da década de 40, BAILEY et alii 4, 5 , BAKER 6 e HARKEN ${ }^{38,39}$ realizaram operaçōes bem sucedidas para comissurotomia mitral com 0 auxílio de dilatadores mecânicos.

Em 1952, HUFNAGEL 40 implantou a primeira prótese de bola na aorta descendente de um paciente com severa insuficiência aórtica.

No ano seguinte, GIBBON Jr. ${ }^{36}$ desenvolveu, com o auxílio de engenheiros da IBM (International Business Machines Corporation), a máquina de circulação extracorpórea com o respectivo oxigenador,

\footnotetext{
- Da UNICAMP/Campinas e FUNFARME/S. J. Rio Preto, SP, Brasil.

- Do Hospital das Clínicas da UNICAMP. Campinas, SP.

*.. De Braile Cardiocirurgia. São José do Rio Preto, SP.

*. De Braile Biomédica. São José do Rio Preto, SP.

Endereço para correspondência: Av. Juscelino Kubitschek 3101 CEP: 15091-450 - São José do Rio Preto, SP, Brasil. Tel.: (0172) 274988 -

Fax (0172) 273177
} 
BRAILE, D. M.; VOLPE, M. A.; RAMIN, S. L.; SOUZA, D. R. S. - Tratamento cirúrgico das valvopatias. Parte 1. Rev. Bras. Cir. Cardiovasc., 9 (2): 113-122, 1994

abrindo não só as cavidades cardiacas para o cirurgião, como também o vasto campo da cirurgia, cardíaca que teve progresso vertiginoso e contínuo desde então.

Em 1956, LILLEHEI et alii ${ }^{45,46}$ realizaram, com sucesso, uma comissurotomia e, posteriormente, no mesmo ano, uma anuloplastia mitral a céu aberto.

No Brasil, ZERBINI et alii ${ }^{71}$, em 1958, introduziram a técnica da comissurotomia mitral sob visão direta, obtendo resultados superiores àqueles da comissurotomia mitral a céu fechado.

$\mathrm{Na}$ década de 50 , ao lado do esforço para a correção cirúrgica das valvas lesadas, avolumaramse também os trabalhos para a criação de um substituto valvular. Nestes estudos, foi possivel distinguir os adeptos de substitutos valvulares mecânicos e os defensores das próteses biológicas 19, 39, 49 .

Foi somente em 1960 que STARR \& EDWARDS 64 substituíram pela primeira vez uma valva mitral por uma prótese de bola, obtendo bons resultados a longo prazo. Esta prótese foi a primeira comercializada em larga escala.

A partir dessa época, o número de válvulas mecânicas foi se tornando cada vez maior e o mundo médico conheceu as válvulas de disco, válvulas de duas cúspides, válvulas de oclusores planos, entre outras.

Entretanto, os pacientes com válvulas cardiacas mecânicas ainda corriam os riscos de apresentar complicações associadas às mesmas, tais como fraturas, deformações, desgastes, vazamentos, deiscência, trombose, hemólise, embolias e infecções. Por esse motivo é que, ao lado das válvulas mecânicas, surgiram também estudos para desenvolvimento de outros tipos de válvulas, como as biopróteses.

Ao se falar em biopróteses, deve-se considerar dois aspectos básicos: biopróteses desenvolvidas com valvas naturais e biopróteses desenvolvidas com tecidos biológicos.

As biopróteses desenvolvidas com valvas naturais compreendem as valvas do próprio paciente (auto-enxerto), as de cadáveres ou receptores de transplantes cardiacos (homoenxertos) e as de animais (heteroenxertos).

O emprego de homoenxertos valvulares iniciouse com MURRAY ${ }^{55}$, que, em 1955, usou uma válvula aórtica fresca em aorta descendente de um paciente portador de insuficiência aórtica grave.

Durante a década de 60 , ROSS 60 e BARRATBOYES ${ }^{7}$ utilizaram enxertos homólogos para substituição da valva aórtica.
Entre nós, a válvula homóloga foi utilizada pela primeira vez em 1967, em posição aórtica, por FELIPOZZI 33 .

As dificuldades na obtenção dos homoenxertos levaram os pesquisadores a desenvolver técnicas que permitissem o uso de tecidos biológicos, que diferem entre si basicamente pelo tipo de tecido empregado.

Existem biopróteses confeccionadas com tecidos retirados do próprio paciente (tecidos autólogos), as de tecidos provenientes de cadáveres (tecidos homólogos) e as de tecidos de animais (tecidos heterólogos).

SENNING 62, em 1966, desenvolveu uma prótese de fascia lata do próprio paciente.

O uso de pericárdio autólogo para confecção de válvulas cardiacas foi introduzido por MORAES et alii 53 (1976).

No início da década de 70 , PUIG et alii 57 desenvolveram uma válvula confeccionada com tecido homólogo, dura mater humana, tratada pelo glicerol.

As próteses heterólogas tiveram um marco histórico com BINET et alii ${ }^{10}$, em 1965 , com a utilização de uma prótese porcina em posição aórtica.

Entretanto, a utilização em larga escala das válvulas heterólogas só foi possivel no final da década de 60 , quando CARPENTIER et alii ${ }^{20}$ (1969) introduziram o uso do glutaraldeido em baixa concentração para a preservação dos tecidos biológicos. Isso propiciou o desenvolvimento de próteses utilizando tecidos animais como a prótese de pericárdio bovino, empregada por IONESCU et alii ${ }^{42}$, em 1971.

No Brasil, também na década de 70 , a válvula de pericárdio bovino tratada pelo glutaraldeído foi pioneiramente difundida por BRAILE et alii 17,18 .

Paralelamente ao esforço no desenvolvimento das biopróteses, houve uma grande evolução das válvulas mecânicas com novos desenhos e a utilização de materiais mais resistentes e menos trombogênicos.

As válvulas de duplo folheto foram introduzidas, em 1977, por EMERY \& NICOLOFF 32 . Também neste ano foram liberadas, para uso clínico, as válvulas de disco basculante Medtronic-Hall 70 .

As próteses de disco basculante Björk-Shiley passaram por grandes mudanças, culminando com a prótese de suporte único, que foi introduzida clinicamente em 1981 13,14.

Atualmente, graças à evolução das técnicas cirúrgicas e da proteção do miocárdio, tem aumen- 
BRAILE, D. M.; VOLPE, M. A.; RAMIN, S. L.: SOUZA, D. R. S. - Tratamento cirúrgico das valvopatias. Parte 1. Rev. Bras. Cir. Cardiovasc., 9 (2): 113-122, 1994

tado o interesse na conservação das valvas, principalmente considerando as complicação que a troca pode acarretar 2, 56,58. Um grande número de cirurgiões tem proposto diferentes técnicas para plástica valvar.

A história da cirurgia das valvas cardíacas continua em franca evolução. O desafio da melhor técnica para restauração da função valvar continua mais presente do que nunca, com dúvidas e questões recentes a serem discutidas e resolvidas, sem que várias das anteriores tivessem sido ainda solucionadas.

\section{INDICAÇÃO CIRÚRGICA}

As indicações básicas para operar as lesões valvares consistem em alívio dos sintomas, prevenção das complicações e da mortalidade.

Em geral os resultados tardios sāo melhores quando as lesões são tratadas em estágios mais precoces, evitando danos irreversiveis à função cardíaca. Entretanto, devemos ponderar que a substituição valvar traz consigo alguns riscos tais como: embolias, trombose, endocardite, deterioração da prótese e complicações da anticoagulação. Isso torna difícil a determinação do momento ideal para a operação e quase sempre optamos por uma indicação um pouco mais precoce do que mais tardiamente. Para as crianças e jovens temos indicado a intervenção cirúrgica mesmo em classe II da NYHA, quando dados ecocardiográficos indicam grande possibilidade da realização de uma plástica.

\section{Estenose Mitral}

1 Sintomas de classe III ou IV da NYHA.

2 Área valvar menor ou igual a $1 \mathrm{~cm}^{2}$.

3 Embolização sistêmica.

4 Estenose mitral moderada e sintomas de classe II da NYHA. Uma operação mais precoce é aconselhável nos casos em que, muito provavelmente, será suficiente uma comissurotomia ou uma plástica mitral 94.

\section{Insuficiência Mitral}

1 Sintomas de classe III ou IV da NYHA.

2 Sintomas de classe II da NYHA com:

a) eixo diastólico menor do ventrículo esquerdo igual ou maior que $6 \mathrm{~cm}$

b) fração de refluxo de $40 \%$ ou mais

c) refluxo mitral $(++++)$ com fração de ejeção menor ou igual a $60 \%$
3 Aumento nos volumes ventriculares ou na fração de refluxo.

4 Qualquer refluxo mitral agudo 54

\section{Estenose Aórtica}

1 Qualquer sintoma devido à estenose (síncope, angina ou insuficiência cardíaca congestiva).

2 Gradiente sistólico máximo maior ou igual a 50 $\mathrm{mmHg}$.

3 Indice valvar menor ou igual a $0,5 \mathrm{~cm}^{2} / \mathrm{m}^{2} 21$.

\section{Insuficiência Aórtica}

1 Sintomas de classe III ou IV da NYHA.

2 Sintomas de classe II da NYHA com:

a) eixo sistólico menor do ventrículo esquerdo igual ou maior que $5,5 \mathrm{~cm}$

b) refluxo de $(+++)$ ou $(++++)$ com fração de ejeção menor ou igual a $50 \%$

3 Aumento dos volumes ventriculares ou diminuição da fração de ejeção.

4 Qualquer refluxo aórtico agudo significativo 54.

\section{Estenose Tricúspide}

Gradiente diastólico médio maior que $5 \mathrm{mmHg}$ ou área valvar tricúspide menor que $2 \mathrm{~cm}^{2} 21$.

\section{Insuficiência Tricúspide}

Não necessita de tratamento cirúrgico na ausência de hipertensão arterial pulmonar. Na presença de hipertensāo arterial pulmonar:

1 Leve e associada a valvopatia mitral reumática: corrigir somente a lesāo mitral.

2 Moderada: anuloplastia.

3 Grave: troca valvar (bioprótese) ${ }^{24}$.

\section{Estenose Pulmonar}

O tratamento cirúrgico é reservado para os casos em que não é possivel a valvoplastia com balão, geralmente valvas displásicas em que há estenose pulmonar grave (pressão sistólica de ventrículo direito maior que $70 \mathrm{mmHg}$ ou gradiente VD-AP maior que $60 \mathrm{mmHg})^{25}$.

\section{Insuficiência Pulmonar}

Indica-se cirurgia para os casos de insuficiência 
primária isolada, em que temos intratável insuficiência cardiaca sendo preconizada a troca valvar pulmonar 25 .

\section{Endocardite Infecciosa Ativa}

1 Critérios menores para intervenção cirúrgica ${ }^{26}$ :

a) insuficiência cardíaca melhorada com medicação

b) embolia isolada

c) vegetação inequívoca do lado esquerdo do coração

d) fechamento mitral precoce (pode não ocorrer na insuficiência mitral associada)

e) rotura de corda tendínea

f) endocardite protética precoce exceto se causada por Streptococcus muito sensivel à penicilina

g) endocardite tricúspide por gram negativo;

h) febre persistente sem causa aparente

i) aparecimento de sopro regurgitante em prótese aórtica

j) não disponibilidade de antibiótico apropriado bactericida

2 Critérios maiores para intervenção cirúrgica: 26

a) insuficiência cardiaca progressiva

b) insuficiência cardíaca significativa

c) episódios embólicos múltiplos d) bacteremia persistente

e) endocardite por fungos

f) corpo estranho extravalvular

g) aparecimento de bloqueio de ramo, bloqueio AV ou pericardite purulenta

h) deiscência ou obstrução de válvula protética;

i) recidiva após tentativa "adequada".

A cirurgia na endocardite infecciosa pode ser indicada por um sistema de classificação por pontos (Tabela 1).

\section{CONDUTA PRÉ-OPERATÓRIA}

A avaliação pré-operatória dos valvopatas tem por finalidade apreciar a história clínica, exame fisico, eletrocardiograma, radiografia de tórax, ecocardiograma, estudo hemodinâmico, teste ergométrico, estudo radioisotópico e exames laboratoriais (eletrólitos, hemograma, coagulograma, anti-HIV, sorologia para hepatite e urina tipo I).

As necessidades hidricas variam com a idade, de $3 \mathrm{ml} / \mathrm{kg} / \mathrm{h}$ no recém-nascido a $1,5 \mathrm{ml} / \mathrm{kg} / \mathrm{h}$ no adulto ${ }^{3}$.

A hidratação dos pacientes é calculada pelo peso corpóreo. Em crianças, a hidratação respeita os seguintes critérios: até $10 \mathrm{~kg}$ - $100 \mathrm{ml} / \mathrm{kg} / \mathrm{dia}$; de

TABELA 1

SISTEMA DE CLASSIFICAÇĀO POR PONTOS PARA INDICAÇÃO CIRÜRGICA NA ENDOCARDITE INFECCIOSA*

\begin{tabular}{|c|c|c|}
\hline \multirow[t]{2}{*}{ DISTÚRBIO } & \multicolumn{2}{|c|}{ ESCALA DE PONTOS } \\
\hline & $E V N$ & $E P$ \\
\hline \multicolumn{3}{|l|}{ - Insuficiência cardíaca } \\
\hline Grave & 5 & 5 \\
\hline Moderad & 3 & 5 \\
\hline Leve & 1 & 2 \\
\hline - Etiologia fúngica & 5 & 5 \\
\hline - Bacteremia persistente & 5 & 5 \\
\hline - Microorganismo diferente do Streptococcus sensivel & 1 & 2 \\
\hline - Recorrência & 2 & 3 \\
\hline Um episódio maior de embolia & 2 & 2 \\
\hline Dois ou mais êmbolos sistêmicos & 4 & 4 \\
\hline - Vegetaçōes detectadas pelo ecocardiograma & 1 & 1 \\
\hline - Fechamento mitral precoce pelo ecocardiograma & 2 & N/A \\
\hline - Rotura das cordoalhas tendinosas ou músculos papilares & 3 & N/A \\
\hline - Bloqueio cardiaco & 3 & 3 \\
\hline Rotura do seio de Vasalva ou septo interventricular & 4 & 4 \\
\hline - Instabilidade de prótese & $N / A$ & 5 \\
\hline - Endocardite protética precoce (<60 dias) & N/A & 2 \\
\hline - Regurgitação periprotética & N/A & 2 \\
\hline
\end{tabular}

* Acúmulo de 5 ou mais pontos sugere necessidade de troca valvar. $\mathrm{N} / \mathrm{A}=$ não aplicável; $\mathrm{EVN}=$ endocardite de valva nativa; $\mathrm{EP}=$ endocardite protética. COUTO \& OLIVEIRA ${ }^{24}$. 
BRAILE, D. M.; VOLPE, M. A.; RAMIN, S. L.; SOUZA, D. R. S. - Tratamento cirúrgico das valvopatias. Parte 1. Rev. Bras. Cir. Cardiovasc., 9 (2): 113-122, 1994

10 a $20 \mathrm{~kg}$ - $1000 \mathrm{ml}$ mais $50 \mathrm{ml} / \mathrm{kg}$ acima dos 10 $\mathrm{kg}$; acima de $20 \mathrm{~kg}-1500 \mathrm{ml}$ mais $20 \mathrm{ml} / \mathrm{kg}$ acima dos $20 \mathrm{~kg}{ }^{1}$. Habitualmente a hidratação é feita com soro glicosado a $5 \%$ e soro fisiológico a $0,9 \%$ na proporção 2/3 : 1/3; contendo $20 \mathrm{mEq}$ de potássio por litro 65 .

Nas crianças, a desidratação e a hipoglicemia merecem atenção particular, pois estas não toleram grandes variações hídricas e glicêmicas.

A reposição hídrica no paciente adulto deve considerar as perdas basais, bem como as possiveis perdas extraordinárias. Geralmente a hidratação é feita com $2 \mathrm{ml} / \mathrm{kg} / \mathrm{h}$, tendo como líqüido de escoIha a solução de Ringer simples.

Em relação ao jejum, é adotada a seguinte medida: de 0 a 6 meses - 4 horas; de 6 meses a 3 anos - 6 horas e acima de 3 anos - 8 horas de jejum 3 .

Antes de serem encaminhados ao Centro Cirúrgico, os pacientes são pesados, medidos e submetidos a tricotomia do tórax, abdomen e membros, sendo posteriormente lavados criteriosamente com povidine degermante (cerca de 1 hora antes).

A medicação pré-anestésica deve propiciar sedação adequada. Doses excessivas dessas medicações são perigosas, pois esses pacientes são extremamente sensiveis a opiáceos e hipnóticos potentes.

Em crianças menores que um ano, a medicação pré-anestésica de escolha é a cetamina, nas maiores é habitual o uso de midazolam. Nos adultos são preferidos os benzodiazepínicos (diazepan $10 \mathrm{mg}$ ou midazolan $15 \mathrm{mg}$ ) administrados por via oral, cerca de 1 hora antes de serem encaminhados ao Centro Cirúrgico ${ }^{3}$.

\section{PRÓTESES VALVULARES CARDIAACAS}

Nos últimos 30 anos tem sido observado um grande avanço no desenvolvimento das próteses valvulares cardíacas. Os substitutos atuais funcionam bem, mas cada um deles traz consigo algumas desvantagens. A variedade das próteses disponiveis atesta a incapacidade de qualquer modelo existente preencher as necessidades de uma prótese ideal.

As características dos substitutos valvulares norteiam em determinados casos a seleção de uma prótese em preferência a outras. A escolha da prótese deve ser individualizada para cada paciente.

As características de uma válvula ideal são: a) boa hemodinâmica; b) ausência de problemas tromboembólicos; c) durabilidade; d) resistente à infecção; e) ausência de hemólise; f) inerte, atóxica; g) fácil de introduzir; h) nenhuma interferência nas outras estruturas; i) acessivel economicamente; j) companhia fabricante fidedigna; k) bom controle de qualidade; I) silenciosa ${ }^{54}$. $O$ interessante é que muitos destes principios já haviam sido estabelecidos, por Harken, no início dos anos 60 (HARKEN et alii ${ }^{39}$ ).

Existem duas grandes classes de próteses valvulares: a) mecânicas: bola e gaiola (StarrEdwards, Cutter), disco basculante (Björk-Shiley, Medtronic-Hall, Lillehei-Kaster, Omniscience) e duplo folheto (St. Jude Medical); b) teciduais (biopróteses): válvulas de pericárdio (lonescu-Shiley, Carpentier-Edwards) e válvulas de porco (Hancock, Carpentier-Edwards).

As válvulas biológicas confeccionadas no Brasil são Biocor, Labcor, Braile-Biomédica, Flumen, Bioval, Fisics, Cardioprótese e H.P., entre outras.

\section{Válvulas mecânicas}

\section{Tipos de válvulas}

a) Starr-Edwards: esta foi a primeira prótese mecânica utilizada com sucesso em posição mitral, introduzida clinicamente em 1960. Seu posicionamento é fácil, mas exige um espaço relativamente grande devido à gaiola, desvantagem em pacientes com ventrículo esquerdo pequeno e/ ou aorta estreita ${ }^{59}$. Com uma função hemodinâmica satisfatória, exceto em pequenos tamanhos, esta prótese é a única mecânica que praticamente nāo tem ruído. Os gradientes transvalvulares são maiores que o das próteses de disco e de duplo folheto do mesmo tamanho 51,52 .

b) Bjork-Shiley: introduzida em 1969, esta prótese passou por muitas mudanças até culminar com a Monostrut (válvula com suporte único), largamente usada na atualidade. Com a parte fixa construída por uma única peça de metal e um disco convexo-côncavo de carbono pirolítico, essa prótese abre-se em ângulo de $70^{\circ}$, fornecendo fluxo quase igual de ambos os lados do disco. Não há relatos de fratura ou falha deste último modelo ${ }^{11}$. Esta válvula não é silenciosa, sendo seu desempenho hemodinâmico superior ao das próteses de Starr, particularmente para os pequenos tamanhos 12 .

c) St. Jude Medical: clinicamente implantada pela primeira vez em 1977, esta prótese tem um baixo perfil e estrutura inteiramente de carbono pirolítico, com dois discos semicirculares que se abrem em ângulo de $85^{\circ}$. Esta válvula apre- 
senta um grande orifício útil e excelente função hemodinâmica ${ }^{29}$. Seus gradientes de pressão são os menores associados a quaisquer das próteses mecânicas disponíveis. A tendência à trombose valvular 8,28 e o ruído são menores quando comparados às válvulas de disco basculante.

d) Medtronic-Hall: inicialmente implantada em 1977, é uma válvula de disco basculante que usa um bastão-guia através de uma abertura no centro do disco. Suas características de fluxo são ligeiramente melhores quando comparada com a válvula Björk-Shiley. Isso relaciona-se ao fato de o disco abrir-se em maior ângulo $\left(70^{\circ}\right.$ para a mitral e $75^{\circ}$ para a aórtica) nas primeiras 70 .

\section{Complicações mais freqüentes das válvulas mecânicas}

a) falha estrutural: as próteses atuais foram progressivamente aperfeiçoadas e têm uma durabilidade que teoricamente seria por toda vida, com taxas despreziveis de falhas estruturais $9,11,22,28$. O risco de complicações mecânicas é constante durante o tempo.

b) trombose valvular: atualmente a trombose valvular tem uma baixa incidência, variando de 0 a menos de $1 \%$ por ano $22,28,63,69$, quando analisadas atuarialmente.

c) tromboembolismo: as próteses mecânicas têm um risco aumentado de tromboembolismo bem como uma incidência definida de sangramento associado à anticoagulação ${ }^{70}$. Diante de uma anticoagulação adequada, as taxas de tromboembolismo variam de 0,5 a $3 \%$ por paciente-ano para a válvula aórtica 31 e de 0,5 a $5 \%$ por paciente-ano para a válvula mitral 70 . As próteses mecânicas atuais parecem ter riscos de tromboembolismo semelhantes, desde que seja feita uma anticoagulação efetiva ${ }^{70}$. $\mathrm{O}$ controle adequado da anticoagulação continua sendo um dos grandes problemas associados à utilização das próteses mecânicas. A anticoagulaçāo eficiente situa-se entre dois extremos representados pelo tromboembolismo e pela hemorragia. Nenhum paciente com válvula mecânica pode prescindir de anticoagulação.

\section{Válvulas de tecidos biológicos}

\section{Tipos de válvulas}

a) heteroenxerto de porco

As válvulas internacionalmente mais conhecidas são a Hancock e a Carpentier-Edwards, ambas fixadas pelo glutaraldeído. Os heteroenxertos de porco apresentam um fluxo central. A hemodinâmica é satisfatória nos tamanhos médio e grande, entretanto, os menores diâmetros podem produzir gradientes importantes em pacientes adultos 21, 23, 68 . Este tipo de bioprótese apresenta baixos índices de complicações tromboembólicas, o que permite a supressão da anticoagulação a longo prazo ${ }^{70}$. A incidência de trombos em pacientes não anticoagulados é equivalente à dos pacientes com válvulas mecânicas anticoagulados 47 .

\section{b) heteroenxerto de pericárdio bovino}

O perocárdio, preservado pelo glutaraldeído, é montado sobre um suporte, geralmente flexivel, permitindo uma área de fluxo central maior que a observada pelos heteroenxertos de porco. Isso poderia justificar o seu desempenho hemodinâmico superior principalmente nos diâmetros menores.

A durabilidade e a ocorrência de eventos tromboembólicos são comparáveis aos encontrados nos heteroenxertos de porco ${ }^{41}$. Este tipo de bioprótese também apresenta alterações degenerativas aceleradas em jovens 17,70

Mundialmente, as válvulas de pericárdio bovino mais conhecidas são a Ionescu-Shiley e a CarpentierEdwards.

\section{Complicações mais freqüentes das biopróteses}

a) falha estrutural: a incidência de falhas primárias tem seu risco aumentado com o tempo, o principal problema é a degeneração tecidual causada pela calcificação, sendo particularmente alto em crianças e jovens $17,35,37,43,47$. Entre os jovens, a bioprótese em posição mitral é mais afetada que a aórtica, contudo, no grupo de 30 a 59 anos, o risco de falha é o mesmo para ambas e significativamente menor para as aórticas em pacientes mais idosos 43 .

As próteses em posição mitral têm um agravante ao trauma por serem submetidas a uma pressão mais elevada durante a sistole. Por outro lado, as próteses de pequeno diâmetro, em posição aórtica, ficarão expostas a um maior grau de estresse ${ }^{34}$.

A degeneração geralmente envolve um aumento gradual da disfunção, o que permite a reoperação em caráter semi-eletivo $35,37,48$.

b) tromboembolismo: o uso das próteses biológicas reduz a taxa de tromboembolismo, mas não - elimina. A doença valvar, particularmente a mitral, por si só expõe o paciente ao risco de acidentes tromboembólicos. A incidência de embolia varia de 0,2 a $3,8 \%$ por ano para a válvula aórtica e 0,3 a $5,1 \%$ por ano para a 
válvula mitral 70 . As taxas de trombose são baixas quando comparadas com as válvulas mecânicas 17,70 . A maioria dos pacientes não necessita de anticoagulação crônica, bastando - uso de antiagregantes plaquetários 17 .

Os estudos randomizados não chegaram a uma conclusão sobre qual seria a melhor prótese cardiaca: se biológica ou mecânica. Os fatores relacionados aos pacientes provavelmente são os responsáveis pela maior parte das complicaçōes, que, no entanto, são imputadas à válvula 17, 70 . Existem Serviços que preconizam a anticoagulação de pacientes em fibrilaçāo atrial, com embolias prévias e com átrio esquerdo aumentado, mesmo utilizando biopróteses. Parece que este grupo especifico de pacientes apresenta mais problemas embólicos $44,58,70$.

\section{Seleção de uma prótese valvular cardíaca}

A seleção de uma prótese valvular depende de duas características principais:

\section{Fatores relacionados a procedimentos cirúrgicos}

As variações anatômicas são importantes na seleção de uma prótese cardiaca. Deve-se avaliar - anel valvar, utilizando a maior válvula possivel, uma vez que os orifícios protéticos são significativamente menores que os naturais. As válvulas mecânicas têm maior área de orifício que as biopróteses para o mesmo tamanho de anel. Tal fato, associado ao conhecimento de que o desempenho hemodinâmico das biopróteses de pequeno tamanho não é bom, justifica a preferência da prótese mecânica para pacientes de anel valvar pequeno 50,70 .

O tamanho da cavidade que receberá a maior parte do volume ocupado pela prótese deve ser considerado. Na posição aórtica, o tamanho da raiz da aorta é determinante, já na posição mitral, o tamanho do ventrículo esquerdo é crítico, principalmente se existir hipertrofia ventricular, o que influencia na escolha de uma prótese de baixo perfil 70 .

\section{Fatores intrinsicos do paciente}

Esses fatores interferem nos resultados a longo prazo e determinam a escolha entre uma bioprótese e uma prótese mecânica ${ }^{44}$. A variável mais importante relacionada aos pacientes é a possibilidade do uso de anticoagulantes, pois isto influencia decisivamente na escolha de uma prótese 12,70 .

A idade tem grande relevância, uma vez que existem diferenças na durabilidade das próteses. De maneira genérica, as válvulas mecânicas são de escolha para pacientes mais jovens e as biopróteses, para pacientes com idade mais avançada. Habitualmente as biopróteses não necessitam de anticoagulação, bastando o uso de anti-agregantes plaquetários $16,17,35,43,69$. Isso é importante em pacientes do sexo feminino e em idade fértil 17,69. Algumas características do paciente podem anular as vantagens de não ser necessária a anticoagulação. Isso ocorre nos casos de lesão mitral, na presença de grande átrio esquerdo, fibrilação atrial e história de embolia sistêmica, uma vez que as complicações tromboembólicas são mais altas neste grupo 44, 57,69.

Um forte indicador das biopróteses é o nivel social e a capacidade do paciente permancer sob terapia anticoagulante a longo prazo $15,17,69$.

Em última análise, deve prevalecer a preferência do paciente, desde que este esteja orientado sobre as vantagens e desvantagens de cada tipo de substituto valvular. Alguns preferem arriscar uma reoperação após troca valvar por prótese biológica a tomar anticoagulante cronicamente com uma prótese mecânica. Outros preferem exatamente o oposto.

\section{RBCCV 44205-239}

BRAILE, D. M.; VOLPE, M. A.; RAMIN, S. L.; SOUZA, D. R. S. - Valvopathies: surgical treatment. Part 1. Rev. Bras. Cir. Cardiovasc., 9 (2): 113-122, 1994.

ABSTRACT: This subject is extensive, and will be divided in three parts. The present paper is related to historic aspects, surgical indication, preoperative approaches and heart valves prostheses. The following two chapters will discuss surgical techniques, reoperations and special condicions like surgical treatment of endocarditis, and bovine pericardial valves.

DESCRIPTORS: heart valves, surgery; valvopathies, history; valvopathies, surgical indications; valvopathies, clinical approach; heart valves prostheses. 
BRAILE, D. M.; VOLPE, M. A.; RAMIN, S. L.; SOUZA, D. R. S. - Tratamento cirúrgico das valvopatias. Parte 1. Rev. Bras. Cir. Cardiovasc., 9 (2): 113-122, 1994

\section{REFERÊNCIAS BIBLIOGRÁFICAS}

1 AMARAL, J. L. G. \& BORDIN, J. O. - Hidratação, reposição volêmica e transfusão. In: SAESP - Sociedade de Anestesiologia do Estado de säo Paulo. Atualização em anestesiologia - 1992. São Paulo, Atheneu, 1992. p. 354-371.

2 ANTUNES, M. J.; WESSELS, A.; SADOWSKI, R. G.; SCHUTZ, J. G.; VANDERDONCK, K. M.; OLIVEIRA, J. M.; FERNANDES, L. E. - Medtronic Hall valve replacement: a third-world population group. J. Thorac. Cardiovasc. Surg., 95: 980-993, 1988.

3 AULER Jr., J. O. C. - Anestesia para tratamento cirúrgico das cardiopatias e circulaçāo extracorpórea. In: SAESP - Sociedade de Anestesiologia do Estado de São Paulo. Atualizaçāo em anestesiologia - 1992. São Paulo, Atheneu, 1992. 212-32.

BAILEY, C. P.; BOLTON, H. E.; REDONDO-RAMIREZ. H. P. - Surgical of the mitral valve. Surg. Clin. N. Am., 32: 1807-1848, 1952

BAILEY, C. P.; O'NEILL, T. J. E.; GLOBER, R. P. JAMISON, W. L.; REDONDO-RAMIREZ, H. P. Surgical repair of mitral insufficiency. Dis. Chest, 19: 125-137, 1951.

6 BAKER, C.; BROCK, R. C.: CAMPBELL, M. Valvulotomy for mitral stenosis: report of six successful cases. Br. Med. J., 1: 1283-1285, 1950.

BARRAT-BOYES, B. G. - Homographt aortic replacement in aortic incompetence and stenosis. Thorax, 19: $131-150,1964$.

BAUDET, E. M.; OCA, C. C.; ROQUES, X. F.; LABORDE, M. N.; HAFEZ, A. S.; COLLOT, M. A. SHIDONI, I. M. - A 5-1/2 year experience with the St. Jude Medical cardiac prosthesis: early and late results of 737 valve replacements in 671 patients. J. Thorac. Cardiovasc. Surg., 90: 137-144, 1985.

9 BEAUDET, R. L.; POIRIER, N. L.; DOYLE, D.; NAKHLE, G.: GAUVIN, C. - The Medtronic-Hall cardiac valve: 7-1/2 years clinical experience. Ann. Thorac. Surg., 42: $644-665,1986$ Shiley heart valve. J. Am. Coll. Cardiol., 6: 1142 1128,1985 monostrut strength. Scand. J. Cardiovasc. Surg., 19: $13-19,1985$

15 BONCHEK, L. I. - Basis for selecting a valve prosthesis. In: MCGOON, D. C. Cardiac Surgery. 2 ed. Philadelphia, FA Davis, 1987. p. 107-125.

16 BORTOLOTTI, V.; MILANO, A.; MAZZUCCO, A.; VALFRÉ, C.; TALENTI, E.; GUERRA, F.; THIENE, G.; GALLUCCI, V. - Results of reoperation for primary tissue failure of porcine bioprosthesis. J. Thorac. Cardiovasc. Surg., 90: 564-569, 1985.

17 BRAILE, D. M. - Prótese valvular de pericárdio bovino: desenvolvimento e aplicação clínica em posiçāo mitral. São Paulo, 1990. [Tese. Doutorado. Escola Paulista de Medicina]. $110 \mathrm{p}$.

18 BRAILE, D. M.; BILAQUI, A.; ARDITO, R. V.; GRECO, O. T.; GARZON, S. A. C.; NICOLAU, J. C.; JACOB, J. L. B.; AYOUB, J. C. A.; FEDOZZI, N. M.; ANGELONI, M. A.; LORGA, A. M. - Bioprótese cardiaca de pericárdio bovino. Arq. Bras. Cardiol., 39: $247-257,1982$.

19. BRAUNWALD, N. S.; COOPER, T.; MORROW, A. G. Complete replacement of mitral valve: successful clinical application of a flexible polyurethane prosthesis. J. Thorac. Cardiovasc. Surg., 40: 1-11, 1960.

20 CARPENTIER, A.; LEMAIGRE, G.; ROBERT, L.; CARPENTIER, S.; DUBOST, C. - Biological factors affecting long-term results of valvular heterografts. J. Thorac. Cardiovasc. Surg., 58: 467-481, 1969.

21 CHAITMAN, B. R.; BONAN, R.; LEPAGE, G. Hemodynamic evaluation of the Carpentier-Edwards porcine xenograft. Circulation, 60: 1170-1175, 1979.

22. COBANOGLU, A.; JAMIESON, W. R. E.; MILLER, D. C.; MCKINLEY, C.; GRUNKEMEIER, G. L.; FOTEN, S.; MIYAGISHIMA, R. T.; TYERS, F. O.; SHUMWAY, N. E.; STARR, A. - A tri-institutional comparison of tissue and mechanical valves using a patient-oriented definition of "treatment failure". Ann. Thorac. Surg., 43: 245-253, 1987.

23 COSGROVE, D. M.; LYTLE, B. W.; WILLIAMS, G. W. - Hemodynamic performance of the CarpentierEdwards pericardial valve in the aortic position in vivo. Circulation, 72: 146-152, 1985.

24 COUTO, A. \& OLIVEIRA, G. - Indicações cirúrgicas nas lesōes tricúspides. In: COUTO, A. A. \& CARNEIRO, R. D. (eds.) Indicações cirúrgicas em cardiologia. Rio de Janeiro, Atheneu, 1991. p. 37-40.

COUTO, A.; OLIVEIRA, G.; CARNEIRO, R. - Lesōes pulmonares. In: COUTO, A. A. \& CARNEIRO, R. D. (eds.) Indicações cirúrgicas em cardiologia. Rio de Janeiro, Atheneu, 1991. p. 41-44.

26 COUTO, A.; RANGEL, F.; CARNEIRO, R. - Indicações cirúrgicas na endocardite infecciosa. In: COUTO, A. 
BRAILE, D. M.; VOLPE, M. A.; RAMIN, S. L.; SOUZA, D. R. S. - Tratamento cirúrgico das valvopatias. Parte 1. Rev. Bras. Cir. Cardiovasc., 9 (2): 113-122, 1994

A. \& CARNEIRO, R. D. (eds.) Indicaçōes cirúrgicas em cardiologia. Rio de Janeiro, Atheneu, 1991. p. 6374.

CUTLER, E. C. \& LEVINE, S. A. - Cardiotomy and valvulotomy for mitral stenosis: experimental observations and clinical notes concerning an operated case with recovery. Boston Med. Surg. J., 188: 1093-1095, 1923.

CZER, L. S.; CHAUX, A.; MATLOFF, J. M.; DEROBERTIS, M. A.; NESSIM, S. A.; SCARLATA, D.; KASS, R. M.; TSAI, T. P.; BLANCHE, C. - Tenyear experience with the St. Jude Medical valve for primary valve replacement. J. Thorac. Cardiovasc. Surg., 100: 44-55, 1990.

CZER, L. S.; MATLOFF, J.; CHAUX, A.; DEROBERTIS, M.: YOGANATHAN, A.; GRAY, R. J. - A 6 year experience with the St. Jude Medical valve: hemodynamic performance, surgical results, biocompatibility and follow-up. J. Am. Coll. Cardiol., 6: 904-912, 1985.

DOYEN, E. - Chirurgie des malformations congenitales ou acquises du couer. Presse Med., 21: 860, 1913.

31 EDMUNDS Jr., L. H. - Thromboembolic complications of current cardiac valvular prostheses. Ann. Thorac. Surg., 34: 96-106, 1982.

32 EMERY, R. W. \& NICOLOFF, D. M. - St. Jude Medical cardiac valve prosthesis: in vitro studies. J. Thorac. Cardiovasc. Surg., 78: 269-274, 1979.

33. FELIPOZZI, H. J. - Transplante de válvulas homólogas no tratamento cirúrgico das lesōes da válvula aórtica. In: Anais do XXIII Congresso Brasileiro de Cardiologia. São Paulo, Sociedade Brasileira de Cardiologia, 1967. p. 37.

34. GABBAY, S.; BORTOLOTTI, U.; WASSERMAN, F.; FACTOR, S. M.; FRATER, R. W. M. - Fatigueinduced failure of the lonescu-Shiley pericardial xenograft in the mitral position: in vivo and in vitro correlation and a proposed classification. J. Thorac. Cardiovasc. Surg., 87: 836-844, 1984.

35. GALLO, I.; NISTAL, F.; ARTINANAO, E. - 6-year to 10year follow-up of patients with the Hancock cardiac bioprosthesis: incidence of primary tissue valve failure. J. Thorac. Cardiovasc. Surg., 92: 14-20, 1986.

GIBBON Jr. H. J. - Applications of a mechanical heart and long apparatus to cardiac surgery. Minn. Med., 37: 171-180, 1954.

37 HAMMOND, G. L.; GEHA, A. S.; KOPF, G. S.; HASHIM S. W. - Biological versus mechanical valves: analysis of 1,116 valves inserted in 1,012 adult patients with a 4,818 patient-year and a 5,327 valve-year followup. J. Thorac. Cardiovasc. Surg., 93: 182-198, 1987
L. R. - The surgical treatment of mitral stenosis. I Valvuloplasty. N. Engl. J. Med., 239: 801-809, 1948.

39. HARKEN, D. E.; SOROFF, H. S.; TAYLOR, W. J. Partial and complete prosthesis in aortic insufficiency. J. Thorac. Cardiovasc. Surg., 40: 744-762, 1960.

40. HUFNAGEL, C. A. \& HARWEY, W. P. - The surgical correction of aortic insufficiency. Bull. Georgetown Med. Cent., 6: 60-65, 1953.

41. IONESCU, M. I. - Long-term durability of the pericardial valve. Z. Kardiol., 75: 207-212, 1986.

42. IONESCU, M. I.; PAKRISHI, B. C.; HOLDEN, M. P. Results of aortic valve replacement with frame supported fascia-lata and pericardial grafts. $J$. Thorac. Cardiovasc. Surg., 64: 340-353, 1972.

43. JAMIESON, W. R. E.; ROSADO, L. J.; MUNRO, A. I.; GEVERN, A. N.; BURR, L. H.; MIYAGISHIMA, R. T.; JANUSZ, M. T.: TYERS, G. F. - Carpentier-Edwards standard porcine bioprosthesis: primary tissue failure (structural valve detereoration) by age groups. Ann. Thorac. Surg., 46: 155-162, 1988.

44. KHURI, S. F.; FOLLAND, E. D.; SETHI, G. K.; SOUCHEK, J.; OPRIAN, C.; WONG, M.; BURCH FIEL, C.; HENDERSON, W. G.; HAMMERMEISTER, K. E. - Six month postoperative hemodynamics of the Hancock heterograft and the Björk-Shiley prosthesis: results of a Veterans Administration Cooperative prospective randomized trial. J. Am. Coll. Cardiol., 12: $8-18,1988$

45. LILLEHEI, C. W.; GOTT, W. L.; DEWALL, R. A.; VARCO, R. L. - Surgical correction of pure mitral insufficiency by annuloplasty under direct vision. Lancet, 77: 446449, 1957

46. LILLEHEI, C. W.; GOTT, W. L.; DEWALL, R. A.; VARCO, R. L. - The surgical treatment of stenotic or regurgitation lesions of the mitral and aortic valves by direct vision utilising a pump-oxygenator. - $J$. Thorac. Cardiovasc. Surg., 85: 154-190, 1958.

47. MAGILLIGAN Jr. D. - Porcine bioprostheses. In: CRAWFORD Jr., F. A., (ed.) Current heart valve prostheses. Philadelphia, Hanley \& Belfus, 1987. p. 269.

48. MAGILLIGAN, D. J.; LEWIS, J. W.; JARA, F. M. Spontaneous degeneration of porcine bioprosthetic valves. Ann. Thorac. Surg., 30: 259-259, 1980.

49 MERENDINO, K. A. (ed.) - Prosthetic valves for cardiac surgery. Springfield,Thomas. 1961.

50 MILANO, A. P.; BORTOLOTTI, U.; MAZZUCCO, A.; GUERRA, F.; MAGNI, A.; GALLUCCI, V. - Aortic valve replacement with the Hancock standard, BjörkShiley and Lillehei-Kaster prostheses. J. Thorac. Cardiovasc. Surg., 98: 37-47, 1989. 
Performance characteristics of the Starr-Edwards model 6120 aortic valve prosthesis beyond ten years. J. Thorac. Cardiovasc. Surg., 88: 193-207, 1984.

MILLER, D. C.; OYER, P. E.; STINSON, E. B.; REITZ, B. A.; JAMIESON, S. W.; BAUMEARTNER, W. A.; MITCHELL. R. S.: SHUMWAY, N. E. - Ten to fifteen year reassessment of the performance characteristics of the Starr-Edwards model 6120 mitral valve prosthesis. J. Thorac. Cardiovasc. Surg., 85: 1-20, 1983.

53. MORAES, C. R.; ARRUDA, M. B.; LAGRECA, J. R.; ESCOBAR, M.; CAVALCANTI, J. L.; VICTOR, E. G.; RAPOSO, L.; GOMES, J. M. P. - Válvulas cardiacas de fascia-lata, dura mater e pericárdio (resultados em 55 casos operados). Rev. Col. Bras. Cir., 3:6062, 1976.

54. MORGAN, R. J.; DAVIS, J. T.; FRAKER, T. D. - Estado atual das próteses valvulares. In: TARANTO, G.; SANTOS, R. S.; FERREIRA, S. M. (trads.) Clinicas cirúrgicas da América do Norte: progressos recentes em cirurgia cardiaca. Rio de Janeiro, Interamericana, 1985. 725-747.

55. MURRAY, G. - Homologous aortic valve segment transplants as surgical treatment for aortic and mitral insufficiency. Angiology, 7: 466-471, 1956.

56. OLESEN, K. H.: RYGG, I. H.; WENNEVOLD, A.; NYBOE, J. - Long-term follow-up in 185 patients after mitral valve replacement with the Lillehei-Kaster prosthesis: overall results and prosthesis-related complication. Eur. Heart J., 8: 680-688, 1987.

57. PUIG, L. B.; VERGINELLI, G.; BELLOTTI, G.; KAWABE, L.; FRACK, C. C. R.; PILEGGI, F.; DECOURT, L. V.; ZERBINI, E. J. - Homologous dura mater cardiac valves: preliminary study of 30 cases. J. Thorac. Cardiovasc. Surg., 64: 154-160, 1972.

58. REUL. G. J.; COOLEY, D. A.; DUNCAN, J. M.; FRAZIER, O. H.; HALLMANN, G. L.; LIVESAY, J. J.; OTT, D. A.; WALKER, W. E. - Valve failure with IonescuShiley bovine pericardium bioprosthesis: analysis of 2680 patients. J. Vasc. Surg., 2: 192-204, 1985.

59. ROBERTS, W. C. - Chosing a substitute cardiac valve: type, size, surgeon. Am. J. Cardiol., 38: 633-644, 1976.
60. ROSS, N. - Homograft replacement of the aortic valve. Lancet, 2: 487, 1962.

61. SAMWAYS, D. W. - Cardiac peristalsis: its nature and effects. Lancet, 1: 927, 1898.

62. SENNING, A. - Aortic valve replacement with fascialata. Acta. Chir. Scand., 356B: 17-20, 1966.

63. STAREK, P. J. K.; BEAUDET, R. L.; HALL, K. V. - The Medtronic-Hall valve: development and clinical experience. In: CRAWFORD Jr., F. A. (ed) Current heart valve prostheses. Philadelphia, Hanley \& Belfus, 1987. p. 223.

64. STARR, A. \& EDWARDS, M. L. - Mitral replacement: clinical experience with a ball valve prosthesis. Ann. Surg., 15: 726-740, 1961.

65. STEWARD, D. J. - Manual of pediatric anesthesia. New York, Churchill Livingstone, 1985. p. 30.

66. SOUTTAR, H. J. - The surgical treatment of mitral stenosis. Brit. Med. J., 2: 603-606, 1925.

67. TUFFIER, T. - État actuel de la chirurgie intrathoracique. In: Transactions International Congress Medicine, 17. London, 1913. p. VII-316.

68. UBAGO, J. L.; FIGUEROS, A.; COLMAN, T. Hemodynamic factors that affect calculated orifice areas in the mitral Hancock xenograft valve. Circulation, 61: 394-398, 1980.

69. VALLEJO, J. L.: GONZALEZ-SANTOS, J. M.; ALBERTOS, J.; RIESGO, M. J.; BASTIDA. M. E.; RICO, M. J.; GONZALEZ-DIEGO, F.; ARCAS, R. Eight year's experience with the Medtronic-Hall valve prosthesis. - Ann. Thorac. Surg., 50: 429-436, 1990.

70. WERNLY, J. A. \& CRAWFORD, M. H. - Escolhendo uma prótese valvular cardiaca. In: ARAÚJO, M. A. C. \& LANA, R. L. (trads.) Clínicas cardiológicas: doença cardiaca valvular. Rio de Janeiro, Interlivros, 1991. p. $353-362$.

71. ZERBINI, E. J.; BITTENCOURT, D.; JATENE, A. Surgical treatment of 150 cases of mitral valves lesion under extra-corporeal circulation. Mal. Cardiovasc., 4: $473-483,1963$. 